\title{
ASPECTOS HISTÓRICOS, ECOLÓGICOS Y SOCIALES ASOCIADOS A Salix humboldtiana EN LA RIBERA DEL RÍO AGRIO EN LA PATAGONIA ARGENTINA
}

\author{
Dezzotti, Alejandro ${ }^{5}$; Sbrancia, Renato ${ }^{5}$; Attis Beltrán, Hernán ${ }^{5}$; \\ Velásquez, $\mathrm{Abel}^{5}$ y Mortoro Ariel $^{5}$
}

\section{RESUMEN}

Salix humboldtiana, el único sauce nativo de América del Sur, conforma la fase sucesional temprana en ambientes riparios de zonas húmedas y semiáridas, desde el norte de México y hasta el norte de la Patagonia en la Argentina y Chile. En el límite meridional de la distribución, la especie está representada por individuos aislados o formando pequeños grupos que representarían el relicto de una distribución más amplia.

En este estudio se analizó la presencia histórica de $S$. humboldtiana en el norte de la Patagonia argentina, basada en documentos que datan desde el siglo. 18. Además, en la ribera del río Agrio inferior $\left(69^{\circ} 42^{\prime}-70^{\circ} 08^{\prime} \mathrm{O}, 38^{\circ} 18^{\prime}-38^{\circ} 29^{\prime} \mathrm{S}\right)$ se evaluó i) la estructura y el poder calorífico de $S$. humboldtiana basado en un censo poblacional y la densidad de la madera, respectivamente, ii) la abundancia de otras plantas leñosas nativas y exóticas, a partir de datos de incidencia presencia/ausencia y iii) la percepción de los pobladores y el uso actual de plantas leñosas basado en una encuesta.

En el área del río Agrio inferior, el clima es semiárido frío, la temperatura y precipitación total media anual son de $13,8{ }^{\circ} \mathrm{C}$ y $163,5 \mathrm{~mm}$, respectivamente, el balance hídrico medio anual es $48,0 \mathrm{~mm}$ y el índice de aridez es 6,9. La densidad de $S$. humboldtiana se estimó en 3,6 ind/ha, el $75 \%$ de los árboles tenía un diámetro $<5 \mathrm{~cm}$ y el $86 \%$ tenía menos de 10 años. El $17,4 \%$ de los pobladores presentaban necesidades básicas insatisfechas, y el consumo y la existencia de leña se estimaron en $2.750 \mathrm{~m}^{3}$ /año y $32.290 \mathrm{~m}^{3}(0,35 \%$ de $S$. humboldtiana), respectivamente. El $77 \%$ de los entrevistados indicó que la abundancia actual de $S$. humboldtiana era menor y que la de leñosas exóticas aumentó.

Hasta mediados del siglo 20, S. humboldtiana habría sido un árbol frecuente en la margen de los ríos patagónicos, a juzgar por la recurrente mención en documentos históricos. Sin embargo, estas referencias no permiten asumir que la población era continua y extensa. El norte de la Patagonia representa el límite austral de la distribución natural, cuyo clima árido, mediterráneo y continental restringiría el desempeño ecológico de $S$. humboldtiana.

A escala regional, la invasión biológica, la hibridación con plantas exóticas, el uso no sustentable y la transformación riparia causada por la urbanización, la agricultura, la extracción de áridos y la actividad hidroeléctrica constituirían factores antropogénicos de degradación y destrucción de la vegetación formada por $S$. humboldtiana. Esta situación justifica su inclusión al marco que brindan las leyes de protección ambiental de bosques naturales. Sin embargo, es necesario además implementar programas de desarrollo y conservación en los cuales las dimensiones social y ecosistémica sean claves.

Palabras clave: Salix humboldtiana, distribución histórica, estructura poblacional, conservación de bosques, especies leñosas exóticas.

\footnotetext{
${ }^{5}$ Grupo de Ecología y Manejo de Sistemas Forestales, Universidad Nacional del Comahue, Sede San Martín de Los Andes, Pasaje de la Paz 235, Q8370AQA San Martín de los Andes, Argentina. adezzotti@gmail.com.
} 


\section{SUMMARY}

Salix humboldtiana, the only willow native to South America, forms the early successional phase of riparian environments in humid and semi-arid regions, from northern Mexico to northern Patagonia in Argentina and Chile. At the southern limit of the geographical range, S. humboldtiana is represented by individuals isolated or forming small groups, which would represent the remnant of a broader distribution.

In this study, the historical presence of $S$. humboldtiana in northern Patagonia from Argentina was analysed, based on documents dating from the century.18. Furthermore, in the waterside of the lower Agrio river $\left(69^{\circ} 42^{\prime}-70^{\circ} 08^{\prime} \mathrm{W}, 38^{\circ} 18^{\prime}-38^{\circ} 29^{\prime} \mathrm{S}\right)$, were analysed i) the structure and calorific value of $S$. humboldtiana based on a census and the density of the wood, respectively, ii) the abundance of other native and exotic woody plants from presence / absence, incidence data, and iii) the perception of the inhabitants and the current use of woody plants based in a survey.

In the lower Agrio river area, the climate is cold and semi-arid, the mean annual temperature and total precipitation are $13.8^{\circ} \mathrm{C}$ and $163.5 \mathrm{~mm}$, respectively, the mean water balance is $-48.0 \mathrm{~mm}$ and the aridity index is 6.9 . Tree density was estimated in $3.6 \mathrm{ind} / \mathrm{ha}, 75 \%$ of $S$. humboldtiana trees exhibited $<5 \mathrm{~cm}$ diameter, and $86 \%$ showed less than $10 \mathrm{yr}$. old. The $17.4 \%$ of the residents had unsatisfied basic needs, the consumption and the existence of firewood were estimated in $2,750 \mathrm{~m} 3 / \mathrm{yr}$ and $32,290 \mathrm{~m} 3(0.35 \%$ of $\mathrm{S}$. humboldtiana), respectively. The $77 \%$ of people indicated that at present, the abundance of $S$. humboldtiana was lower and that of exotic woody species increased.

Until the mid 20 century, $S$. humboldtiana would have been a frequent plant on the margin of the Patagonian rivers, judging by the recurrent mention in historical documents. However, these references do not allow the assumption that the population was continuous and extensive. Northern Patagonia represents the southern limit of the natural distribution of $S$. humboldtiana, whose arid, Mediterranean and continental climate would restrict its ecological performance.

At regional scale, biological invasion, hybridization with exotic plants, unsustainable use, and riparian transformation caused by urbanization, agriculture, mineral extraction, and hydroelectric activity would constitute anthropogenic factors of degradation and destruction of the vegetation formed by $S$. humboldtiana. This situation justifies the inclusion of this species under the laws that specifically protect natural forests. However, it is also necessary to implement development and conservation programs in which the social and ecosystem dimensions represent key issues.

Key words: Salix humboldtiana, historical distribution, population structure, forest conservation, exotic woody species. 


\section{INTRODUCCIÓN}

Las especies de Salix (Salicaceae) tienen una enorme relevancia global en el contexto de la silvicultura sostenible y el desarrollo rural (Kuzovkina et al., 2008). Este género está compuesto por unas 400 especies, de las cuales solo el sauce de plata del Cabo (Salix mucronata Thunb.) y el sauce criollo (Salix humboldtiana Willd.) son nativos del hemisferio sur (Argus, 1997). Salix humboldtiana es el único sauce nativo de América del Sur, y se extiende desde el norte de México, en Durango y Nuevo León, hasta el norte de la Patagonia, en la IX Región de Chile y la provincia de Chubut en la Argentina (Boelcke, 1986; Batis et al., 1999; Hauenstein et al., 2005). Salix humboldtiana es un árbol siempreverde o caducifolio dioico, presenta reproducción asexual y sexual, polinización entomófila y una semilla ortodoxa que tiene dispersión anemócora e hidrocora, viabilidad breve y germinación epigea (Rodríguez et al., 1983). La especie está categorizada "De preocupación menor" (Barstow, 2018), se la cultiva ampliamente y en algunas áreas se encuentra naturalizada (DEEDI, 2020).

Salix humboldtiana es un árbol sucesional temprano en ambientes riparios de zonas húmedas y semiáridas (Parolin et al., 2002; Barstow, 2018). En la región tropical tiende a ser reemplazado por árboles tolerantes a la sombra, mientras que en la templada no (Parolin et al., 2002; Caso et al., 2010; Thomas y Leyer, 2014). El régimen hidrológico regula la sucesión, a través del cual se forman bancos que promueven la colonización durante la fase de sedimentación, que se erosionan eliminando la vegetación durante la fase de inundación.

La regeneración ocurre en forma episódica en estos bancos cuando el nivel hidrométrico no causa inundación o sequía extensas. Los árboles de mayor tamaño contribuyen a este proceso porque disminuyen la velocidad del agua y el transporte de sedimentos (Lewis y Franceschi, 1979; Liotta, 2001; Thomas y Leyer, 2014). La reproducción vegetativa es menos dependiente de estas condiciones y las plantas que se originan de este modo presentan mayor sobrevivencia (Moggridge y Gurnell, 2009).

En el actual territorio argentino, desde finales del Pleistoceno $S$. humboldtiana suministró bienes asociados al tronco y la corteza, rama, hoja y flor. Las sociedades cazadoras-recolectoras utilizaban la madera para combustible y confeccionar herramientas y flechas (Rodríguez, 2000; Brea et al., 2001, 2014; Colobig, 2012). Desde el siglo17, su utilización era intensa a juzgar por un bando de 1610 que prohibió la corta en Buenos Aires (López, 1886). La especie era usada para la fabricación de embarcaciones, viviendas y objetos, y para elaborar forraje y medicina (Casamiquela, 1985; Di Sapio y Gattuso, 1994; Borodowski y Suárez, 2004; Prates, 2009; Larroulet et al., 2011).

En la Patagonia argentina, en la actualidad S. humboldtiana está representada por individuos aislados o en pequeños grupos, que representarían el relicto de una distribución más amplia que disminuyó debido a la actividad humana (Hunziker, 1992; Shafroth et al., 1994; Cremer, 2003; Birken y Cooper, 2006; Natale et al., 2008, 2012, 2018; Budde et al., 2011; Thomas et al., 2012; Datri et al., 2013; Thomas y Leyer, 2014; Bozzi et al., 2014).

\section{OBJETIVOS}

El objetivo general de este estudio fue desarrollar conocimiento ecológico, social y tecnológico sobre $S$. humboldtiana para contribuir a diseñar estrategias de conservación in- y exsitu, y su incorporación al marco de protección que brindan la Ley 26.331 de "Presupuestos mínimos de protección ambiental de los bosques nativos" (MJDH, 2020) y la Ley 2.780 de "Ordenamiento territorial de bosques nativos la provincia de Neuquén" (LPN, 2020). 
Los objetivos particulares fueron analizar la presencia histórica de $S$. humboldtiana en el norte de la Patagonia argentina, las características y el consumo de leña de los pobladores, y la estructura, el crecimiento y el estado de conservación de $S$. humboldtiana en un área particular de la provincia de Neuquén.

\section{MATERIALES Y MÉTODOS}

\section{Área de Estudio}

El área de estudio se localizó en la ribera sur del río Agrio inferior, desde el paraje Bajada Vieja y hasta la desembocadura con el río Neuquén $\left(69^{\circ} 42^{\prime}-70^{\circ} 08^{\prime} \mathrm{O}, 38^{\circ} 18^{\prime}-38^{\circ} 29^{\prime} \mathrm{S}\right)$, tiene aproximadamente 900 ha y está conformada por una longitud de $60 \mathrm{~km}$ y un ancho medio de 150 $\mathrm{m}$ (Figura $\mathrm{N}^{\circ}$ 1). El río Agrio nace en el lago Caviahue en la cordillera de los Andes, y luego de recorrer $250 \mathrm{~km}$ desemboca en el río Neuquén. Este río se une con el río Limay y forman el río Negro, que desemboca en el océano Atlántico después de atravesar aproximadamente $600 \mathrm{~km}$. El relieve del área de estudio es suave y está dominado por mesetas escalonadas, terrazas fluviales, llanuras aluviales, depresiones con ocasionales lagunas y salinas, y sierras bajas. Los suelos del valle son Aridisoles cálcicos y Entisoles torriortentes franco arenosos, mientras que en las partes elevadas dominan los afloramientos rocosos (Pereyra y Bouza, 2019).

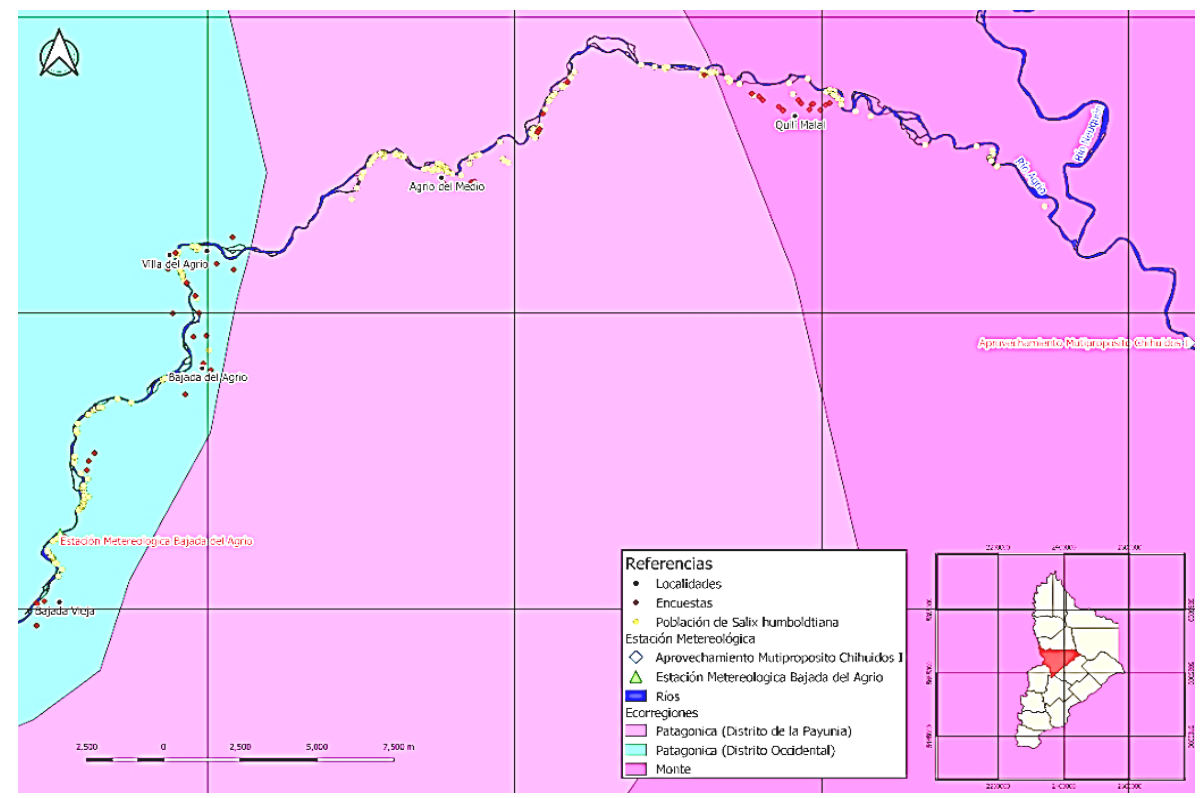

Figura $\mathrm{N}^{\circ} 1$

IMAGEN DE LA LOCALIZACIÓN DE Salix humboldtiana EN LA RIBERA SUR DEL RíO AGRIO INFERIOR EN LA PROVINCIA DE NEUQUÉN EN LA ARGENTINA 
El área de estudio pertenece a las Ecorregiones Patagónica (Distritos Occidental y de la Payunia) y del Monte (Monte Austral). La vegetación es graminoso-arbustiva, las plantas dominantes son xerófilas y la cobertura aérea es baja.

En el Distrito del Monte Austral se encuentra típicamente Larrea spp. (Zygophyllaceae), en el de la Payunia el solupe (Ephedra ochreata Miers. (Ephedraceae) y la chilladora (Chuquiraga rosulata Gaspar. Asteraceae), y en el Occidental Festuca spp. (Poaceae) (Oyarzábal et al., 2018) (Figura $\mathrm{N}^{\circ}$ 1).

La actividad productiva se caracteriza por el autoconsumo, la tecnología sencilla y los límites prediales flexibles, y comprende la ganadería bovina, ovina y caprina, y la producción de aves, forraje, hortalizas, legumbres, cereales y frutas (Bendini y Alemany, 2004).

En esta área se encuentran las localidades Bajada del Agrio (884 habitantes) y Quili Malal (184) (DPEC, 2010), y los parajes Agrio del Medio, Villa del Agrio, Bajada del Puente y Bajada Vieja.

En el río Neuquén, cerca de la confluencia con el río Agrio, se planifica construir el Aprovechamiento Multipropósito Chihuido I, cuyo embalse de 18.600 ha inundará el área de estudio (EMHIDRO, 2013) (Figura $\mathrm{N}^{\circ}$ 1).

\section{Metodología}

\section{- $\quad$ Aspectos Históricos y Sociales}

La presencia pasada de $S$. humboldtiana en el norte de la Patagonia argentina se basó en documentos y crónicas de personas que se desplazaron por la región por diferentes motivos y circunstancias desde finales del siglo 18.

Estas fuentes históricas brindaron información sobre $S$. humboldtiana en las cuencas de los ríos Colorado, Limay, Negro y Chubut, y algunos de sus afluentes. (Molina, 1805; de la Cruz, 1806; Villarino, 1837; Lorentz y Niederlein, 1883; Hauman, 1913; d'Orbigny, 1945; Cabrera, 1953; Roig, 1955; Moreno, 1969; Darwin, 2009).

El conocimiento sobre las características de los pobladores y las propiedades, el consumo, el abastecimiento y la procedencia de la leña y madera, y el conocimiento de la especie, se basó en una encuesta que involucró 22 preguntas organizadas en 5 categorías.

Esta encuesta fue respondida por residentes en Quili Malal (14 personas), Bajada del Agrio (9), Agrio del Medio (3), Villa del Agrio (3), Bajada del Puente (3) y Bajada Vieja (3) $(n=35)$ (Cuadro $\mathrm{N}^{\circ} 1$ ). 
- Características del poblador

Cantidad de miembros del hogar

¿Desde cuándo reside en la zona?

Actividad o empleo

- Características de la propiedad

Localización

Tamaño del lote

Uso $o$ actividad del lote

- Condición de la propiedad (propia, alquilada, otra)

¿Tiene ganado? ¿Cuántos animales de cada tipo de ganado?

- Demanda y abastecimiento de leña y madera ¿Qué artefactos tiene para producir calor? (ej., cocina a leña) ¿Qué combustible utiliza para la cocina, la calefacción, el agua caliente y el alumbrado

¿Prefiere alguna especie para uso de leña?, ¿cuál?

¿Utiliza S. humboldtiana?, ¿para qué?

- Procedencia de la madera y leña

Provisión del estado provincial (¿cuánto?, ¿cuándo?)

Recolectada (¿cuánto?,¿dónde?, ¿distancia?)

Comprada (¿dónde?, ¿cuánto?, ¿cuánto dinero gastó durante este año?, ¿cuánto incide este gasto en la economía familiar?, periodicidad de compra).

- Conocimiento de S. humboldtiana

¿Conoce a S. humboldtiana?, ¿lo puede identificar?

¿Sabe dónde se encuentra?

¿Sabe si había más S. humboldtiana en el pasado?

¿Sabe qué uso le daban a $S$. humboldtiana en el pasado?

¿Sabe si existe alguna planta que en el pasado era poco abundante o no existía y ahora es muy abundante?

¿Estaría dispuesto a plantar S. humboldtiana en su propiedad?, ¿qué superficie?, ¿tiene agua para riego?

\section{- $\quad$ Aspectos Ecológicos y Productivos}

El clima se basó en información sobre el valor medio mensual de la precipitación total $(P)$, temperatura $(T)$ y la humedad relativa $(H R)$ colectada entre 1999 y 2019 en la estación meteorológica Bajada del Agrio (38²7' 14,47" S y 705' 10,04" O, 646 msnm.) (AIC, 2020) (Figura $\left.\mathrm{N}^{\circ} 1\right)$.

El clima se clasificó de acuerdo a Köppen-Geiger (Peel et al., 2007). La evapotranspiración potencial mensual $(E T p)$ y el balance hídrico climático mensual $(B H)$ (Thornthwaite y Matter, 1957) se determinaron de acuerdo a: 


$$
\begin{gathered}
E T p=16\left(\frac{10 T}{\sum I_{c}}\right)^{a} \frac{N_{s}}{12} \frac{d}{30} \\
B H=P m-E T p
\end{gathered}
$$

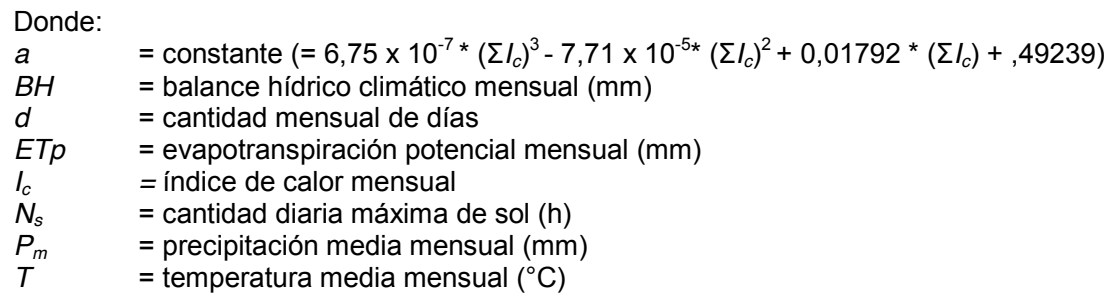

Para esta estación se calculó el índice de aridez mensual de de Martonne (1926) de acuerdo a:

$$
I_{M}=\frac{12 P}{T+10}
$$

Donde:

$$
\begin{array}{ll}
I_{M} & =\text { índice de aridez } \\
P & =\text { precipitación total media mensual }(\mathrm{mm}) \\
T & =\text { temperatura media mensual }\left({ }^{\circ} \mathrm{C}\right)
\end{array}
$$

Si $I_{M}>40$ el clima es húmedo, $I_{M}=30$ - 40 es subhúmedo, $I_{M}=20$ - 30 es semiárido, $I_{M}=10-20$ es árido, $I_{M}=5-10$ es subdesértico y $I_{M}=0-5$ es desértico.

Los individuos de $S$. humboldtiana fueron categorizados en juveniles (altura total $h<1,3$ m) y adultos $(h \geq 1,3 \mathrm{~m})$. Cada árbol fue georeferenciado (GPS), y a los adultos se le midió el diámetro a la altura del pecho ( $D A P, 1,3 \mathrm{~m}$ del suelo) (cinta diamétrica, forcípula). Una muestra de árboles adultos se seleccionó de tal manera de involucrar todas las clases de tamaño $(n=33)$, se les midió el $D A P$ (cinta diamétrica) y $h$ (hipsómetro láser), se tomó una muestra de barreno (barreno tipo Pressler) y se le contó los anillos anuales para la determinación de la edad $(e)$ y el crecimiento diamétrico $(c d)$.

En el gabinete las muestras se procesaron y analizaron a través del procedimiento de Fritts (1982) (lupa 24x). La estimación poblacional de $h$ y $e$ se basó en la construcción de funciones de regresión de estas variables con el DAP (ANOVA, $\mathrm{p}<0,05)$.

La estimación del volumen total se basó en un modelo lineal con los datos obtenidos de la cubicación de árboles en pie $(n=25)$. A estos árboles se les midió el $D A P$ (forcípula), se les estimó el diámetro a diferentes alturas (ocularmente) y las alturas total, de inicio de copa y de las bifurcaciones para estimar la longitud de las trozas y el fuste (hipsómetro láser). La calidad de la estimación ocular se evaluó a través de una submuestra de los valores estimados en comparación con los valores medidos, y no se observaron diferencias significativas (prueba de $t, \mathrm{p}=0,922, \mathrm{n}=$ 29). La cubicación de la troza basal se realizó con la fórmula de Huber y de los ápices con la del cono; el resto con la fórmula de Smalian (Cailliez, 1980).

En el área de estudio se instalaron en forma sistemática 36 transectas de $50 \mathrm{~m}$ cada una ,en forma paralela a la dirección del valle fluvial, separadas entre sí aproximadamente a $600 \mathrm{~m}$. En 
cada transecta se localizó un punto de muestreo cada $1 \mathrm{~m}$, para determinar la frecuencia de plantas leñosas nativas y exóticas, utilizando el método del punto de intercepción $(n=1.800)$ (Kent, 2011).

La frecuencia basada en datos de incidencia presencia/ausencia permite cuantificar la abundancia de plantas que no están adaptadas a mediciones apropiadas de densidad, biomasa, uso de energía o cobertura, teniendo en cuenta las limitaciones que surgen para establecer el límite físico de cada individuo (Jost et al., 2010).

Se evaluó la frecuencia de las especies leñosas jarilla macho (Larrea cuneifolia Cav.), jarilla hembra (Larrea divaricata Cav.), jarilla crespa (Larrea nitida Cav.), Populus spp. (Salicaceae), rosa mosqueta (Rosa rubiginosa L. Rosaceae), Salix exóticos, S. humboldtiana, molle blanco (Schinus johnstonii F.A.Barkley Anacardiaceae) y tamarisco (Tamarix ramosissima Ledeb. Tamaricaceae).

La relación entre la existencia y el consumo de leña se basó en información sobre el tamaño de la población de $S$. humboldtiana, la cantidad de hogares (DPEC, 2010) y el consumo de leña (encuesta) de Bajada del Agrio y Quili Malal.

La existencia de leña de $S$. humboldtiana y de las demás especies se basó en estimaciones volumétricas y en la frecuencia relativa de $S$. humboldtiana respecto del total de especies leñosas, respectivamente.

\section{- $\quad$ Aspectos Tecnológicos}

El poder calorífico de la madera de $S$. humboldtiana se lo comparó con el del árbol del paraíso (Elaeagnus angustifolia, L. Elaeagnaceae), el fresno blanco americano (Fraxinus americana L., Oleaceae), S. johnstonii, el sauce mimbre (Salix fragilis L.) y T. ramosissima presentes en el área de estudio.

Se elaboraron probetas de madera con una longitud de $20 \mathrm{~mm}$ de lado, se secaron en forma gradual hasta alcanzar un peso contante (estufa), de acuerdo a la norma ISO 13061-1 (ISO, 2014a), y luego se les determinó el volumen $\left(v_{0}\right)$ (calibre, precisión $0,1 \mathrm{~mm}$ ) y peso $\left(m_{0}\right)$ (balanza electrónica, precisión $0,01 \mathrm{~g})$. La densidad de la madera en condición seca $\left(d_{o}\right)$ se estimó de acuerdo a la norma ISO 13061-2 (ISO, 2014b), según:

$$
I_{M}=\frac{m_{\circ}}{v_{o}}
$$

Donde:

$d_{o}=$ densidad de la madera seca $\left(\mathrm{g} / \mathrm{cm}^{3}\right)$

$m_{0} \quad$ = peso de la probeta $(\mathrm{g})$

$v_{0} \quad=$ volumen de la probeta $\left(\mathrm{cm}^{3}\right)$

El número de probetas se determinó para un valor medio del coeficiente de variación de $d_{o}=10 \%$, y los resultados se expresaron de acuerdo a la norma ISO 3129 (ISO, 2012).

El poder calorífico $(p c)$ se estimó de acuerdo al factor de convergencia energética para leña blanda $c e=2.900 \mathrm{kcal} / \mathrm{kg}$ (SEN, 2020), según: 


$$
p c=d_{0} c e
$$

Donde:

$$
\begin{array}{ll}
p c & =\text { poder calorífico }\left(\mathrm{kcal} / \mathrm{m}^{3}\right) \\
d_{o} & =\text { densidad de la madera seca }\left(\mathrm{g} / \mathrm{cm}^{3}\right) \\
c e & =\text { factor de convergencia energética }(\mathrm{kcal} / \mathrm{kg})
\end{array}
$$

\section{RESULTADOS}

\section{Presencia Histórica de S. humboldtiana}

El explorador español Basidio Villarino (1741-1785) realizó un reconocimiento del río Negro en 1782 y 1783, y en su diario de viaje escribió: "Día 6: Al salir el sol proseguí mi viaje, y teniendo espías con la gente casi todo el día en el agua, navegué 3/4 de legua al ONO corregido, y por las vueltas del río 3 leguas. Aquí hay superior terreno en estas rinconadas, y abundante saucería en las islas... Día 24: ...hice el rumbo del NO 1/4 O corregido, 3 millas de distancia. En este intermedio y lo navegado ayer, hay mucha saucería" (Villarino, 1837).

El explorador chileno Justo Molina (1745-1817) durante la recorrida de la ribera sur del río Colorado en el viaje entre Chillán y Buenos Aires en 1804 y 1805, escribió en la actual localidad de Rincón de los Sauces: "...salí de Cubunlubu por la orilla abajo el que corre al Puelche, y camino de arena entre algunas piedras, apartándome algunas vezes del río, y en este día andaria siete leguas; en todo este cajón hay madera de sauce y leña para fuego y muchas lilas que todas tienen pan" (Molina, 1805). El nombre de esta localidad se debería a la presencia de $S$. humboldtiana (Mollo y Vignolo, 2010).

El explorador chileno Luis de la Cruz (1768-1828) en la crónica del viaje entre Concepción en Chile y Buenos Aires en Argentina de 1806, a lo largo del trayecto en las actuales provincias de Neuquén y Río Negro, escribió: "Desde que bajamos a Caycaden, a una y otra parte de la senda, hemos venido dejando lomajes de tierras de las mismas calidades que en él conté. Hay muchos arbustos por todos lados de michis, chacayes, callimamines, colliguayes, quiscos, retamillas, maitenes, y algunos sauces en la orilla del estero de Caycaden...Y siguiendo por el mismo rumbo a las seis cuadras estuvimos en la ribera del poniente del rio Cudileuvu, al abrigo de unos hermosos sauces... Aquí mucho más emboscada de pajas y arbustos chicos, y por ella abajo, ya separándonos del río, ya acercándonos, y en parte por terrenos limpios, y en otras con arbustos con tres y media leguas medidas, llegamos a un bajo que hay bastante saucería..." (de la Cruz, 1806).

El naturalista francés Alcide d'Orbigny (1802-1857) explora el norte de la Patagonia en 1829 y en el diario de viaje escribió: "...el río Negro se parece al río Colorado y al río Sauce, en que desde doce leguas arriba de su desembocadura, hasta muy arriba de su curso, está adornado de sauces, los únicos árboles que crecen naturalmente al sur de Buenos Aires, en todas las pampas" (d'Orbigny, 1945).

El naturalista inglés Charles Darwin (1809-1882) durante el viaje a bordo del HMS Beagle entre 1831 y 1836, en el capítulo sobre el trayecto a través del río Negro el 24 de julio 1833 redactó: "La villa es indiferentemente llamada El Carmen o Patagones. Está edificada frente a un peñón que mira al río, y muchas de las casas han sido excavadas en la arenisca. El río tiene una anchura de 200 a 300 metros y es profundo y rápido. Las numerosas islas, con sus sauces, y los 
farallones salientes, vistos uno tras otro en el límite septentrional del anchuroso valle vestido de verdor, forman, a la brillante luz del sol, un conjunto casi pintoresco" (Darwin, 2009).

El naturalista argentino Francisco Moreno (1852-1919), en el relato de la expedición a la Patagonia de 1876 y 1877 señaló: "...sólo en el valle del Chubut se ven árboles como el sauce (Salix humboldtiana) y una especie de molle muy poco abundante... Exceptuando el sauce colorado indígena (Salix humboldtiana) que según D'Orbigny ha dado el nombre al río Chubut, solo uno que otro eucalipto recién plantado y algunos álamos (Populus dilatata) representan el adorno arbóreo de este valle... " (Moreno, 1969).

Los botánicos argentino - alemán Paul Lorentz (1835-1881) y Gustav Niederlein (18581924), de la Comisión Científica al río Negro de 1879, relataron: "Por primera vez durante la expedición, vimos sauces en las orillas del Sauce Chico, después en los bordes del Río Colorado y del Río Negro" (Lorentz y Niederlein, 1883).

El botánico belga Lucien Hauman (1880-1965) en la expedición al río Negro inferior de 1913 observó la escasez de $S$. humboldtiana y concluyó que, aunque sus semillas eran abundantes y se diseminan adecuadamente, se encontraba entre otras especies cultivadas en una situación de desventaja por un crecimiento más lento (Hauman, 1913).

En 1934, el Instituto Geográfico Militar identificó la región fitogeográfica de $S$. humboldtiana en la cuenca del río Negro (Cabrera, 1953). En 1950, el botánico argentino Fidel Roig (1922-2008) indicó que $S$. humboldtiana predominaba en el bosque en galería de los ríos Negro, Chubut y Limay (Roig, 1955).

\section{Clima}

El clima de Bajada del Agrio es semiárido frío (Bsk).

La temperatura media anual fue $13,8^{\circ} \mathrm{C}(\mathrm{EE}=0,2)(\mathrm{n}=20)$. La temperatura media de los meses cálidos (diciembre, enero y febrero) fue $21,2{ }^{\circ} \mathrm{C}$ y de los fríos (junio, julio, agosto) $7,1{ }^{\circ} \mathrm{C}$, que representó una diferencia de $14,2^{\circ} \mathrm{C}$.

La temperatura media anual máxima y mínima fue $21,4{ }^{\circ} \mathrm{C}(\mathrm{EE}=0,3)$ y $6,2{ }^{\circ} \mathrm{C}(\mathrm{EE}=0,1)$, respectivamente. La temperatura media mensual máxima fue $30,8^{\circ} \mathrm{C}$ en enero y la mínima $0{ }^{\circ} \mathrm{C}$ en julio.

A lo largo del periodo de estudio, la temperatura media mensual máxima absoluta fue $32,7^{\circ} \mathrm{C}$ (enero 2009) y la mínima absoluta fue $-2,5^{\circ} \mathrm{C}$ (agosto 2007), que representó una diferencia de $35,3^{\circ} \mathrm{C}$.

La diferencia entre la temperatura máxima y mínima media mensual fue $15,2{ }^{\circ} \mathrm{C}$ y varió entre $17,6^{\circ} \mathrm{C}$ (febrero) y $12,0^{\circ} \mathrm{C}$ (junio) (Figura $\mathrm{N}^{\circ} 2 \mathrm{~A}$ ).

La precipitación total media anual fue 163,5 mm $(E E=12,3)(n=20)$. La precipitación total media mensual máxima fue $27,4 \mathrm{~mm}$ en mayo y la mínima fue $6,8 \mathrm{~mm}$ en noviembre.

La precipitación total media de los meses cálidos fue $8,5 \mathrm{~mm}$ y de los fríos $18,9 \mathrm{~mm}$. A lo largo del periodo de estudio, el coeficiente de variación medio anual de la precipitación mensual fue $112,0 \%$. 

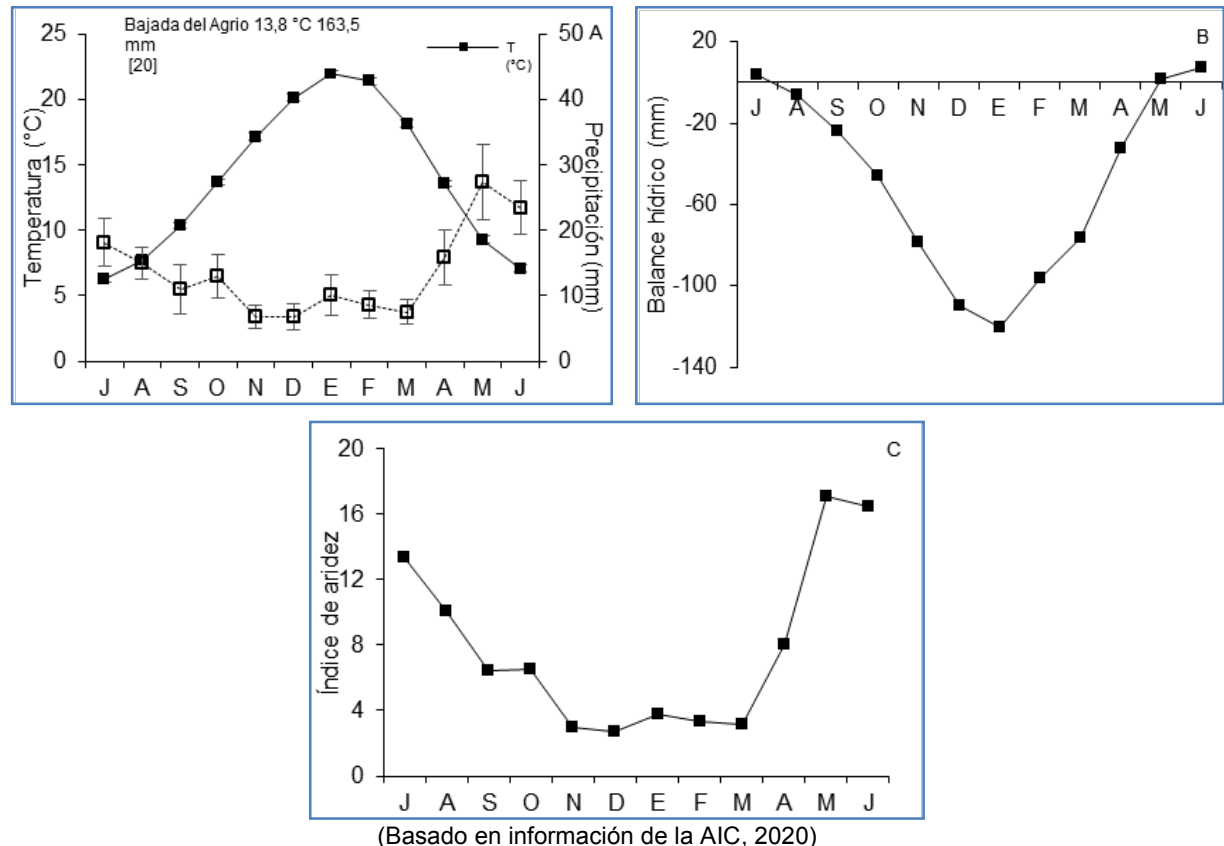

Figura $\mathrm{N}^{\circ} 2$

DIAGRAMA CLIMÁTICO (A), BALANCE HÍDRICO (B) E ÍNDICE DE ARIDEZ (C) DE LA ESTACIÓN METEOROLÓGICA BAJADA DEL AGRIO

La humedad relativa media anual fue $44,7 \%(E E=0,7)$ y varió entre $31 \%$ (diciembre) y $61 \%$ (junio). Entre agosto y mayo existió sequía relativa (Figura $\mathrm{N}^{\circ} 2 \mathrm{~A}$ ).

El balance hídrico promedio fue $-48,0 \mathrm{~mm}(\mathrm{EE}=13,5)$, varió entre $-120,4$ (enero) y 7,4 $\mathrm{mm}$ (junio) y el déficit hídrico se extendió entre agosto y abril (Figura $\mathrm{N}^{\circ} 2 \mathrm{~B}$ ).

El índice de aridez anual fue 6,9 (clima subdesértico) y varió entre 2,7 (diciembre, desértico) y 17 (mayo, árido) (Figura $\mathrm{N}^{\circ} 2 \mathrm{C}$ ).

\section{Estructura de la Vegetación}

En la ribera sur del río Agrio inferior se relevaron 3.281 individuos de $S$. humboldtiana lo que representó una densidad de alrededor de 3,6 ind/ha (Figura $\mathrm{N}^{\circ} 3$ ). La distribución de frecuencias del diámetro se aproximó a una función exponencial negativa (Prueba de KolmogorovSmirnov, $p<0,05)$.

El $74,9 \%$ de los árboles tenían $D A P=<5 \mathrm{~cm}$, el $11,1 \% D A P=5-10 \mathrm{~cm}$, el $9,4 \% D A P=$ $10-20 \mathrm{~cm}$ y el $0,8 \% D A P>40 \mathrm{~cm}$ (Figura $\mathrm{N}^{\circ} 4 \mathrm{~A}$ ). 


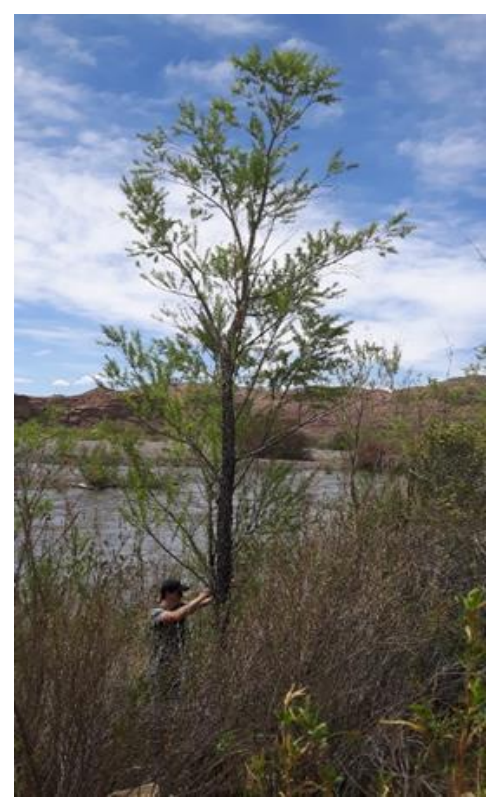

Figura $\mathrm{N}^{\circ} 3$

\section{EJEMPLAR DE Salix humboldtiana EN LA MARGEN DEL RÍO AGRIO}

El $75,7 \%$ de los árboles tenían $h=2-3,9 \mathrm{~m}$ y el 3,7\% tenían $h=12-13,9 \mathrm{~m}$ (Figura $\mathrm{N}^{\circ}$ $4 \mathrm{~B})$. La edad de los árboles se estimó a partir de la relación $D A P=0,4642 e^{1,1542}(\mathrm{~F}=197,2 ; \mathrm{p}<$ 0,$05 ; R^{2}=0,864, n=33$ ) (Figura $N^{\circ} 5 A$ ). De acuerdo a esta estimación, el $86,3 \%$ de los árboles tenía $e=1-9,9$ años y el $1,2 \%$ e $>40$ años (Figura $\left.\mathrm{N}^{\circ} 4 \mathrm{C}\right)$. El $c d=0,819 \mathrm{~cm} /$ año $(\mathrm{EE}=0,052, \mathrm{n}=$ 33).

La altura de los árboles se estimó a partir de la relación $h=2,774 D A P^{0,3742}(\mathrm{~F}=123,6 ; \mathrm{p}$ $<0,05 ; \mathrm{R}^{2}=0,886, \mathrm{n}=32$ ) (Figura $\mathrm{N}^{\circ} 5 \mathrm{~B}$ ). El volumen total de madera y leña de $S$. humboldtiana en el área de estudio se estimó en $113 \mathrm{~m}^{3}$, que representó $0,13 \mathrm{~m}^{3} /$ ha (Figura $\mathrm{N}^{\circ} 5 \mathrm{C}$ ).

En el área de estudio las especies leñosas dominantes nativas son $S$. humboldtiana, $L$. nitida, L. divaricata, L. cuneifolia y $S$. johnstonii, y las exóticas son Populus spp., R. rubiginosa, Salix exóticos y $T$. ramosissima.

El $66,6 \%$ del suelo estuvo cubierto por vegetación, mientras que el suelo desnudo y las piedras involucraron el $32,7 \%$ de la superficie (Figura $\mathrm{N}^{\circ} 6 \mathrm{~A}$ ).

La especie leñosa que exhibió la mayor frecuencia fue T. ramosissima $(17,1 \%)$, mientras que la frecuencia de las especies de Salix exóticos, Larrea spp. y S. humboldtiana fue 7,3\%, 5,0\% y $0,1 \%$, respectivamente. La frecuencia de las especies leñosas nativas y exóticas fue $6,1 \%$ y $25,8 \%$, respectivamente (Figura $\mathrm{N}^{\circ} 6 \mathrm{~B}$ ). 

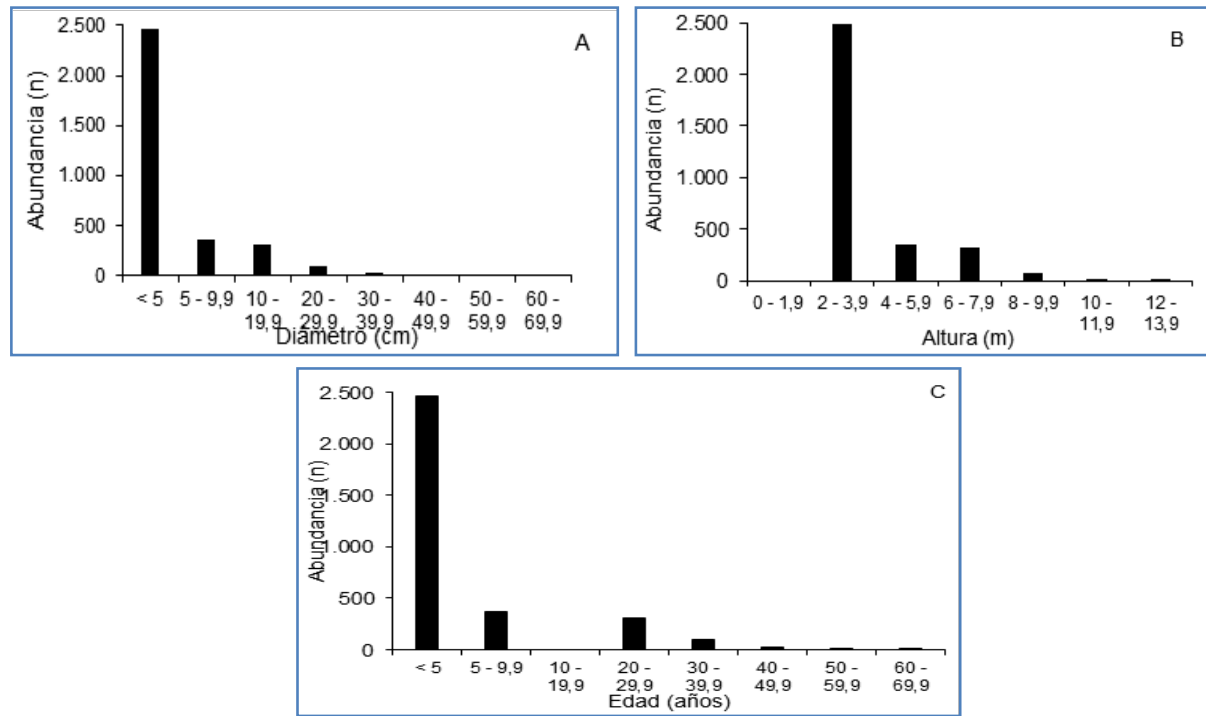

Figura $N^{\circ} 4$

DISTRIBUCIÓN DE LAS FRECUENCIAS DE DIÁMETRO (A), ALTURA (B) Y EDAD (C) DE Salix humboldtiana EN LA RIBERA SUR DEL RÍO AGRIO INFERIOR.
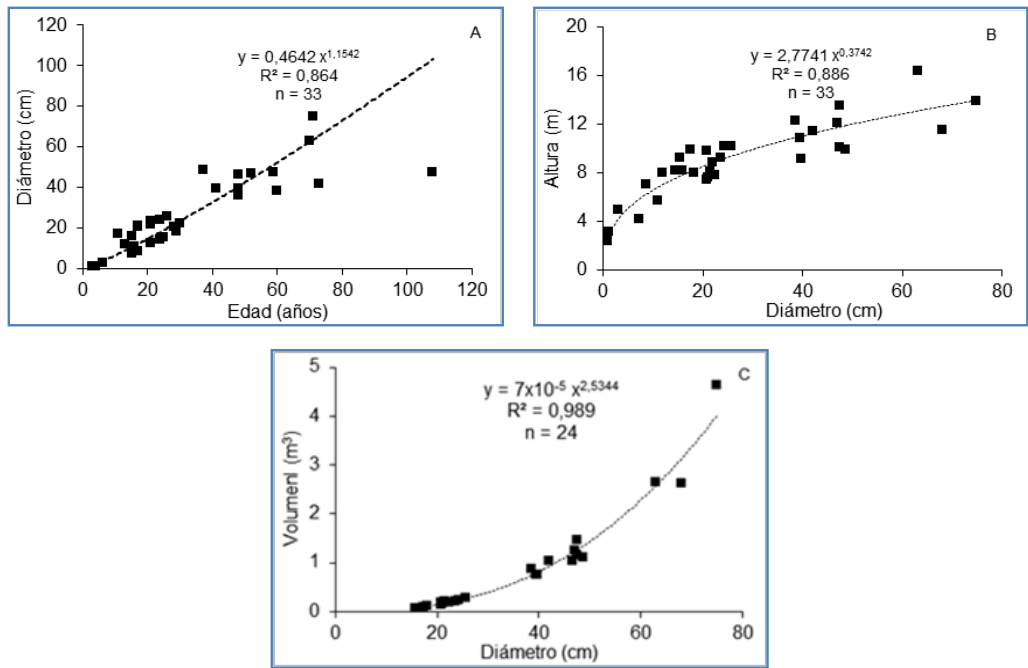

Figura $N^{\circ} 5$

RELACIÓN ENTRE EL DIÁMETRO Y LA EDAD (A), ALTURA (B) Y VOLUMEN TOTAL DE Salix humboldtiana (C) EN LA RIBERA SUR DEL RÍO AGRIO INFERIOR (ANOVA, P < 0,05) 

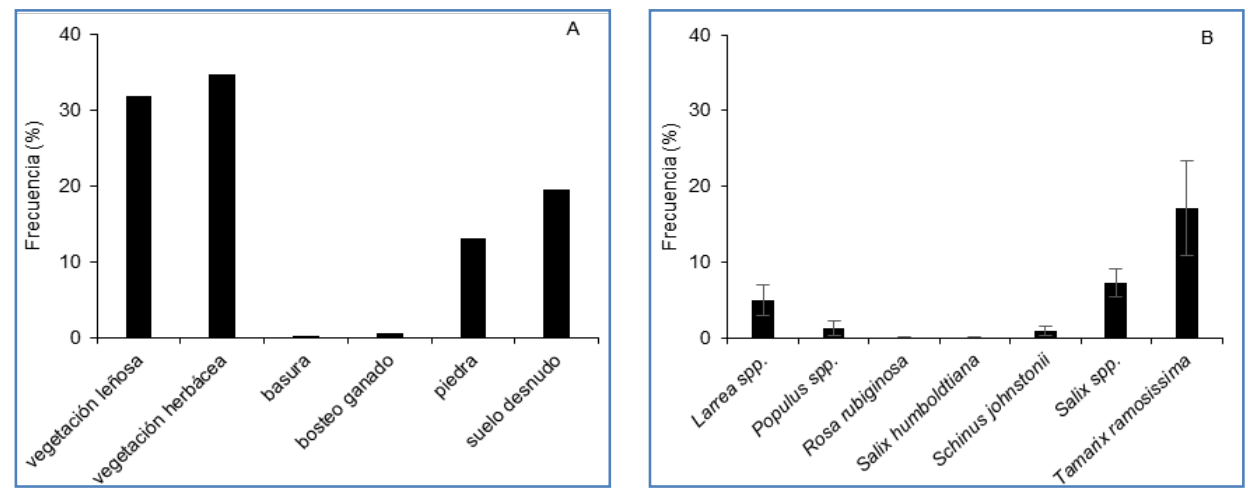

Las barras verticales indican el error estándar de la media $(n=1.800)$

\section{Figura $\mathrm{N}^{\circ} 6$ \\ FRECUENCIA DE COBERTURA DE SUELO (A) Y DE ESPECIES LEÑOSAS (B) EN LA RIBERA SUR DEL RÍO AGRIO INFERIOR.}

\section{Características Sociales}

Las localidades de Bajada del Agrio y Quili Malal tenían 310 hogares con un tamaño medio de 3,4 personas/hogar y de los cuales $17,4 \%$ presentaba necesidades básicas insatisfechas (DPEC, 2010) (Cuadro $\mathrm{N}^{\circ}$ 2).

El $83 \%$ de las personas encuestadas tenían un hogar de hasta 3 miembros, el $80 \%$ residió en el área desde hace más de 40 años y ninguno lo hizo durante menos de 20 años. El 86\% eran productores y empleados estatales. El $71 \%$ eran propietarios del predio o la titularidad se encontraba en proceso de sucesión, $17 \%$ tenían tenencia de la propiedad y $12 \%$ tenían boleto de compra, comodato, contrato o derecho a compra. El $74 \%$ de las propiedades eran de hasta de 5 ha y estaban dedicadas preferentemente a la producción mixta de alfalfa, frutales y hortalizas, $6 \%$ sólo a la ganadería y $3 \%$ estaba combinada con frutales. El $71 \%$ de las viviendas tenía cocina a gas y el $20 \%$ utilizaba además leña, la calefacción era a leña $(86 \%)$, gas $(9 \%)$ o una combinación de ambos (3\%). El agua caliente se basaba en la electricidad (51\%), la leña (34\%) y el gas $(9 \%)$. El $94 \%$ de las viviendas tenía luz eléctrica.

El consumo total de leña se estimó en $2.750 \mathrm{~m}^{3} / a n ̃ o$ y la existencia total de leña se estimó en $32.290 \mathrm{~m}^{3}$, de los cuales el $0,35 \%$ correspondió a $S$. humboldtiana, y la relación

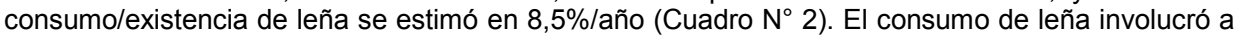
Salix exóticos y Populus (79\%), T. ramosissima (6\%), S. johnstonii (6\%) y S. humboldtiana (3\%). El $62 \%$ de los encuestados no utilizó esta última especie. La leña fue provista por privados (62\%) y el Estado provincial (38\%).

Existen en el área de estudio postes de alambrado de $S$. humboldtiana. Los entrevistados indicaron que en el pasado se la utilizaba frecuentemente para leña, construcción, remedio y postes (Figura $\mathrm{N}^{\circ} 7 \mathrm{~A}$ ). Los restos que datan de 1925 del primer edificio de la Primaria 14 de Quili Malal $\left(38^{\circ} 20^{\prime} \mathrm{S}\right.$ y $69^{\circ} 49^{\prime} \mathrm{O}$ ) conservan los cabios del techo construidos con $S$. humboldtiana (Figura $\mathrm{N}^{\circ} 7 \mathrm{~B}, 7 \mathrm{C}$ ). 
El $97 \%$ de los entrevistados conocían a S. humboldtiana, el $74 \%$ sabían dónde se encontraba actualmente y el $60 \%$ lo plantarían en su propiedad. El $77 \%$ de los entrevistados indicaron que en el pasado $S$. humboldtiana presentaba una superficie y cantidad de individuos mayores y que la distribución de $T$. ramosissima aumentó (Figuras $\mathrm{N}^{\circ} 8$ y $\mathrm{N}^{\circ} 9$ ).

\section{Cuadro $\mathrm{N}^{\circ} 2$ \\ PARÁMETROS UTILIZADOS PARA ESTIMAR LA RELACIÓN ENTRE EL CONSUMO Y LA ABUNDANCIA DE LEÑA EN QUILI MALAL Y BAJADA DEL AGRIO}

\begin{tabular}{|c|c|c|c|}
\hline Variable & & Valor & Fuente \\
\hline Tamaño de la población urbana (ind) & a & 1.066 & \multirow{5}{*}{ DPEC (2010) } \\
\hline Cantidad de hogares $(n)$ & b & 310 & \\
\hline Cantidad de hogares con NBI (n) & c & 54 & \\
\hline Cantidad de hogares con NBI (\%) (c/b) & $d$ & 17,4 & \\
\hline Tamaño del hogar (ind/hogar) (a/b) & $\mathrm{e}$ & 3,4 & \\
\hline Relación $\mathrm{me} / \mathrm{m}^{3}$ & $\mathrm{k}$ & 0,6 & Triana (2001) \\
\hline Cantidad de hogares encuestados ( $n$ ) & $f$ & 23 & \multirow{10}{*}{ Este estudio } \\
\hline Cantidad de hogares encuestados (\%) (f/b*100) & g & 7,4 & \\
\hline Consumo leña encuestados (me/año) & $\mathrm{h}$ & 340 & \\
\hline Consumo leña encuestados $\left(m^{3} / a n ̃ o\right)\left(h^{*} k\right)$ & $\mathrm{i}$ & 204 & \\
\hline Consumo leña encuestados ( $\mathrm{m}^{3} /$ hogar/año) (i/f) & j & 8,9 & \\
\hline Frecuencia relativa de S. humboldtiana (\%) & 0 & 0,35 & \\
\hline Consumo de leña total $\left(\mathrm{m}^{3} / a n ̃ o\right)\left(b^{*} \mathrm{j}\right)$ & I & 2.750 & \\
\hline Existencias totales de leña $\left(\mathrm{m}^{3}\right)\left(\mathrm{m} / \mathrm{o}^{\star} 100\right)$ & $\mathrm{p}$ & 32.286 & \\
\hline Existencias de leña $S$. humboldtiana $\left(\mathrm{m}^{3}\right)$ & $\mathrm{m}$ & 113 & \\
\hline Consumo / existencia de leña (\%) (I/p*100) & $\mathrm{n}$ & 8,5 & \\
\hline
\end{tabular}

me: Metro Estéreo, NBI: Necesidades Básicas Insatisfechas
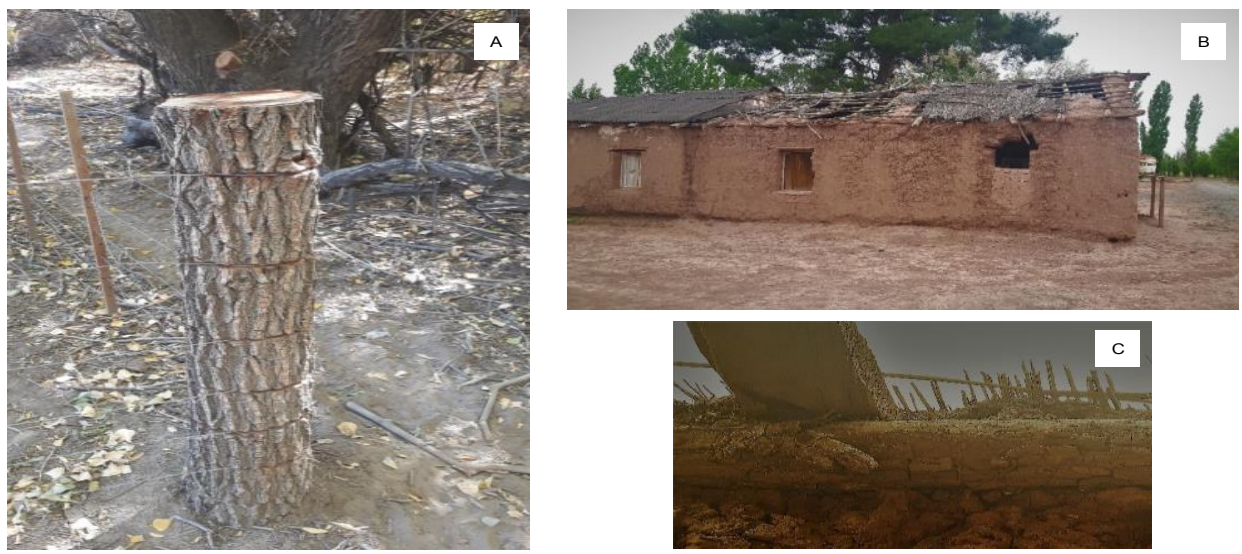

Figura $\mathrm{N}^{\circ} 7$

POSTE (A) Y PRIMER EDIFICIO DE LA ESCUELA PRIMARIA 14 (1925) (B) CON VIGAS DEL TECHO (C) CONSTRUIDOS CON Salix humboldtiana 


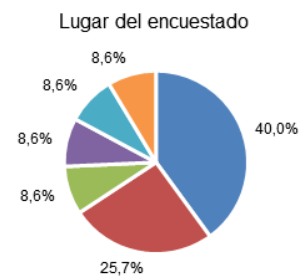

Oficio del encuestado

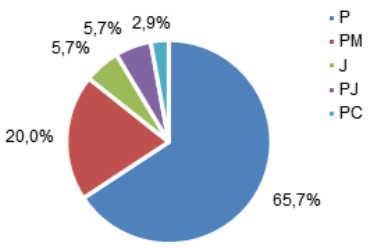

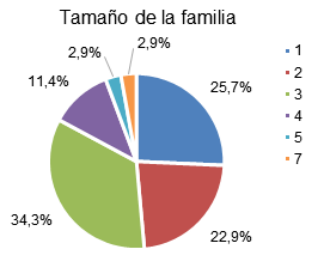

Permanencia (años)

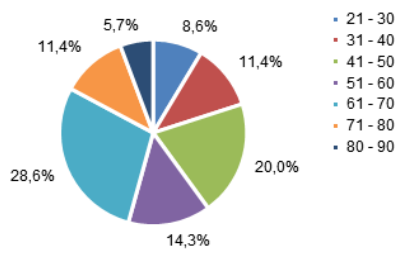

Características del Poblador

AM: Agrio del Medio; BA: Bajada del Agrio; QM: Quili Malal; BP: Bajada del Puente; VA: Villa del Agrio; BV: Bajada Vieja

J: Jubilado/Pensionado; P: Productor; PM: Productor-Empleado; PJ: Productor/Jubilado; PC:

Productor/Criancero
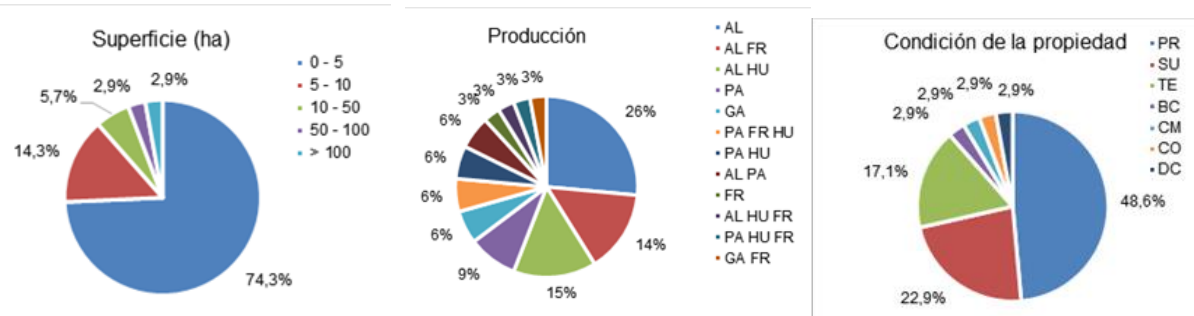

Características de la Propiedad

PR: Propietario; SU: Sucesión; TE: Tenencia; BC: Boleto Compra; CM: Comodato; CO: Contrato;

DC: Derecho a Compra

Figura $\mathbf{N}^{\circ} 8$

ASPECTOS SOCIALES Y PRODUCTIVOS DE LOS POBLADORES

CARACTERÍSTICAS DEL POBLADOR Y CARACTERÍSTICAS DE LA PROPIEDAD (N: 35) 

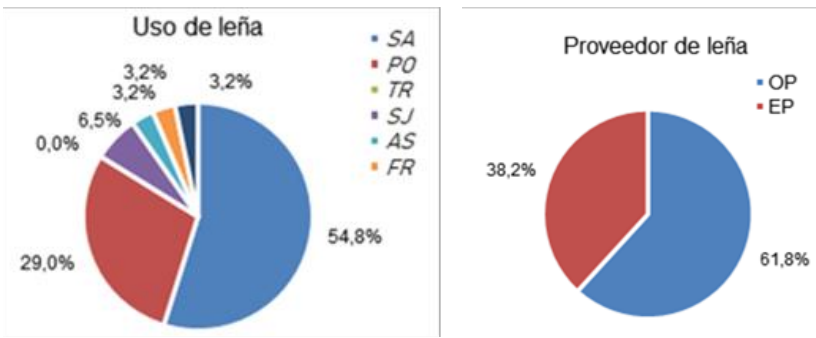

Uso de S. humboldtiana

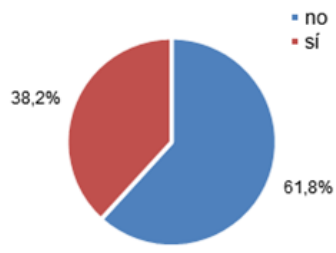

Consumo de Leña

SA: Salix exóticos; PO: Populus spp; TR: T. ramosissima; SJ: S. johnstonii; AS: Acacia; FR: Frutales OP: Proveedor Privado; EP: Estado Provincial
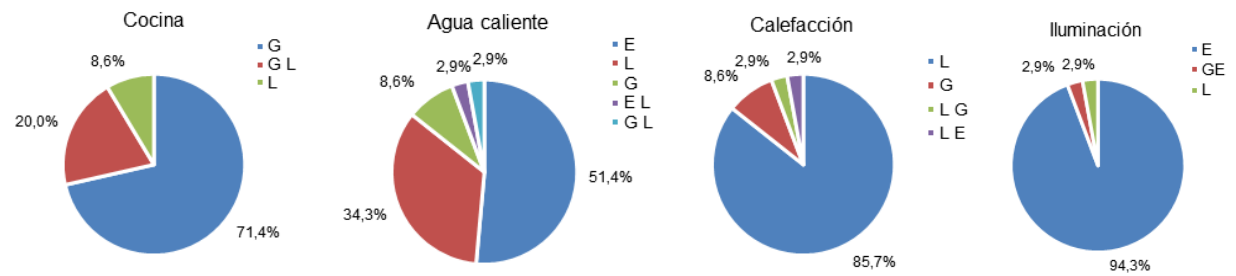

Utilización de Energía

G: Gas; L: Leña; E: Electricidad

¿Reconoce a S humboldtiana?

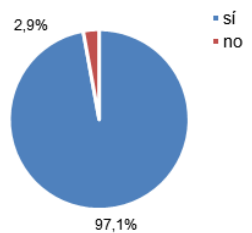

¿Sabe dónde está S. humboldtiana?

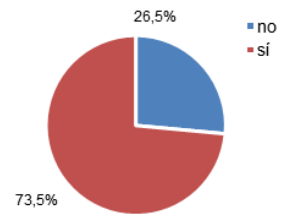

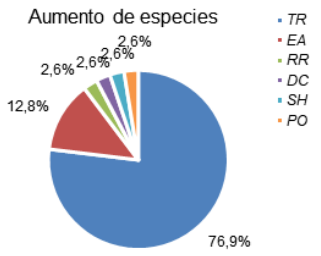

Conocimiento de Especies

TR: T. ramosissima; EA: E. angustifolia; RR: R. rubiginosa; DC: Discaria spp.; SH: S. humboldtiana; PO: Populus spp.

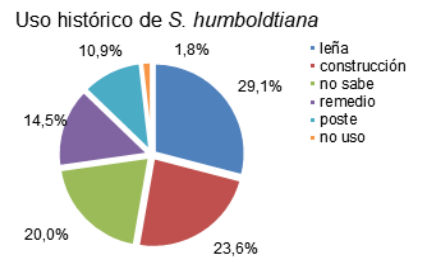

Existencia pasada de S. humboldtiana

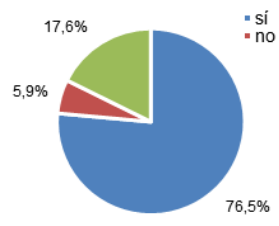

¿Acepta plantar S. humboldtiana?

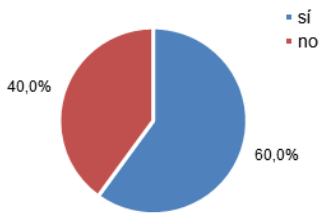

Uso Histórico y Plantación de Salix humboldtiana

Figura $\mathrm{N}^{\circ} 9$

ASPECTOS SOCIALES Y PRODUCTIVOS DE LOS POBLADORES CONSUMO DE LEÑA, UTILIZACIÓN DE ENERGÍA, CONOCIMIENTO DE ESPECIES Y USO HISTÓRICO Y PLANTACIÓN DE Salix humboldtiana ( $\mathrm{N}=35$ ). 


\section{Poder Calorífico de la Madera}

La densidad de la madera difirió significativamente entre especies (ANOVA, Prueba de $\mathrm{LSD}, \mathrm{F}=206,0, \mathrm{p}<0,05, \mathrm{n}=38$ ) y varió entre $0,420(\mathrm{~S}$. humboldtiana, $\mathrm{EE}=0,007, \mathrm{n}=7$ ) y 0,922 $\mathrm{g} / \mathrm{cm}^{3}(T$. ramosissima, $\mathrm{EE}=0,041, \mathrm{n}=6)$ (Figura $\left.\mathrm{N}^{\circ} 10 \mathrm{~A}\right)$. El poder calorífico de la madera varió entre 1,22 (S. humboldtiana) y $2,67 \mathrm{kcal} / \mathrm{m}^{3}$ ( $T$. ramosissima). El poder calorífico de $S$. humboldtiana fue equivalente al de $S$. fragilis y representó el $45,6 \%$ del de $T$. ramosissima (Figura $\left.\mathrm{N}^{\circ} 10 \mathrm{~B}\right)$.
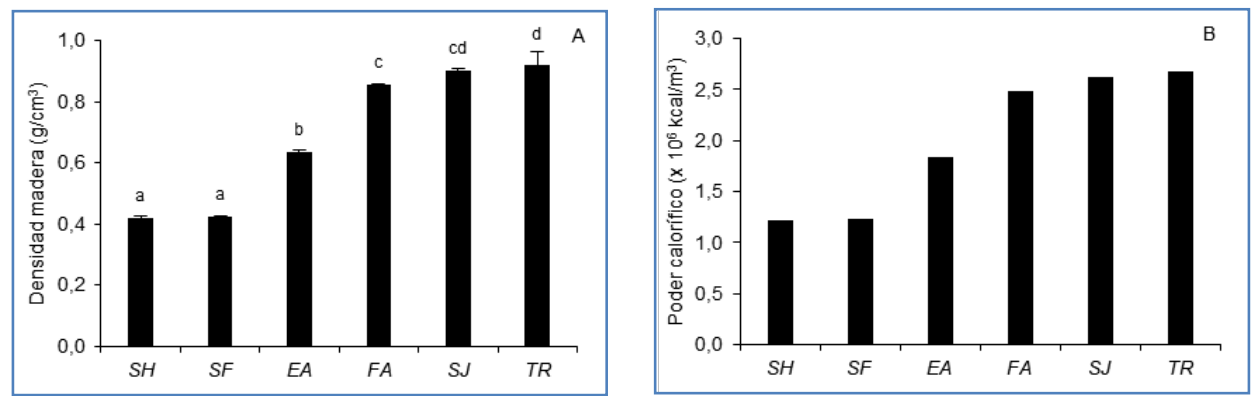

Las barras verticales indican el error estándar de la media y las letras desiguales diferencias significativas entre especies (prueba LSD, $p<0,05$ )

Figura $\mathrm{N}^{\circ} 10$

DENSIDAD (A) Y PODER CALORÍFICO DE LA MADERA (B) DE Salix humboldtiana (SH), Salix fragilis (SF), Elaeagnus angustifolia (EA), Fraxinus americana (FA), Schinus johnstonii (SJ) Y Tamarix ramosissima (TR)

\section{DISCUSIÓN}

La presencia de $S$. humboldtiana en la ribera de los ríos Colorado, Limay, Neuquén, Negro y Chubut fue señalada en numerosas fuentes desde el siglo 18 (Molina, 1805; de la Cruz, 1806; Villarino, 1837; Lorentz y Niederlein, 1883; Hauman, 1913; d'Orbigny, 1945; Cabrera, 1953; Moreno, 1969; Darwin, 2009). Aunque esta información no permite saber si era un árbol de distribución continua, o por el contrario, estaba representado por grupos o individuos aislados, la sola mención indicaría que era frecuente al menos en algunas áreas de ese extenso territorio. Sin embargo, algunos cronistas pudieron haber confundido a $S$. humboldtiana con Salix exóticos, cuyo cultivo e hibridación es anterior a 1860 (Scaramuzzino et al., 2017). Por ejemplo, el naturalista suizo Georges Claraz (1832 - 1930) durante el viaje de 1865 a la región del río Chubut indicó: "...vimos allí también un sauce (trasplantado por el hombre)" (Claraz, 2008).

En la ribera sur del río Agrio existe una escasa cantidad de árboles de $S$. humboldtiana, de los cuales más del $70 \%$ tenía un diámetro menor de $5 \mathrm{~cm}$, una altura de hasta $4 \mathrm{~m}$ y una edad de hasta 10 años. En esta área de estudio, la población reducida y conformada básicamente por individuos jóvenes y pequeños se debería básicamente a factores asociados al clima. El clima presenta una temperatura mínima media mensual entre 0 y $1{ }^{\circ} \mathrm{C}$ y que alcanzó $-2,5{ }^{\circ} \mathrm{C}$ durante algún mes invernal, temperatura máxima media entre 29 y $31^{\circ} \mathrm{C}$, escasa precipitación concentrada en invierno y que en verano no supera los $10 \mathrm{~mm}$ mensuales, y periodos de sequía relativa y déficit hídrico intensos que se extienden prácticamente durante todo el año. 
Este clima semiárido y frío, con influencia continental y mediterránea, determinaría que la dispersión, el establecimiento y el crecimiento de $S$. humboldtiana se restrinja solo a aquellas áreas de ribera con influencia directa del curso de agua. Estas condiciones físicas impondrían restricciones al desempeño espacial y temporal de esta especie, que solo tolera heladas muy suaves y cuyo adecuado desarrollo requiere entre 20 y $40 \%$ de sombra (Belov, 2009).

Los factores antropogénicos que afectan a S. humboldtiana en el ambiente ripario del río Agrio son los mismos que en otras áreas de la Patagonia, aunque con menor intensidad. La invasión biológica que tendría lugar se infiere de la baja frecuencia de $S$. humboldtiana $(0,1 \%)$ en comparación con la de los Salix exóticos y $T$. ramosissima (24,4\%). En este sentido, el $77 \%$ de los pobladores encuestados refieren un aumento de la abundancia de $T$. ramosissima y una disminución de la de $S$. humboldtiana. S. fragilis, Salix alba, Salix babylonica, Salix x rubens y Salix matsudana que presentan altas tasas de crecimiento y colonizan vegetativamente bancos de arena y barrancas de desmoronamiento, que representarían ventajas frente a $S$. humboldtiana que depende de la existencia de sitios de establecimiento más estables (Hunziker, 1992; Shafroth et al., 1994; Borodowski y Suárez, 2004; Budde et al., 2011; Thomas et al., 2012; Thomas y Leyer, 2014). La invasión biológica está asociada además a un proceso irreversible de "dilución genética", debido a que los sauces exóticos se hibridan con $S$. humboldtiana, y luego estas poblaciones híbridas se vuelven a cruzar con los clones introducidos (Bozzi et al., 2014).

En el área de estudio, el uso de leña se basa fundamentalmente en especies leñosas exóticas provistas por proveedores estatales y privados. La utilización de $S$. humboldtiana no es significativa, probablemente porque la abundancia es reducida y porque otras especies leñosas son más abundantes y proveen un mayor contenido energético. Sin embargo, existe un escenario caracterizado por la fragilidad y el riesgo de degradación de S. humboldtiana, asociado a su carácter relictual, a la amplia dependencia de los pobladores en la leña para la cocción, la calefacción y la provisión de agua caliente, la alta proporción de hogares con ingreso mínimo y la escasez general de leña.

La degradación de $S$. humboldtiana es un proceso histórico como se infiere de la opinión de los encuestados acerca del uso pasado frecuente para propósitos energéticos, constructivos y medicinales. Finalmente, el Aprovechamiento Multipropósito Chihuido I inundará las áreas de $S$. humboldtiana del área de estudio (EMHIDRO, 2013). Las represas hidroeléctricas Alicura $\left(40^{\circ} 35^{\prime} \mathrm{S}\right.$

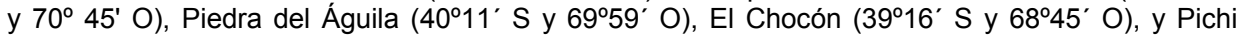
Picún Leufú $\left(40^{\circ} 01^{\circ} \mathrm{S}\right.$ y $\left.69^{\circ} 45,9^{\circ} \mathrm{O}\right)$ han producido cambios en el régimen de los ríos patagónicos: los escasos bancos de arena que se forman en primavera promovieron la regeneración de $S$. humboldtiana que se eliminó durante las crecidas de verano (Bozzi et al., 2014).

\section{CONCLUSIONES}

Hasta mediados del siglo 20, S. humboldtiana habría sido una planta frecuente en la margen de los ríos del norte de la Patagonia argentina en general y del río Agrio en particular. Desde la época colonial, estos árboles están recurrentemente mencionados en las crónicas probablemente no solo porque eran frecuentes, sino además porque contrastaban en el inmenso paisaje natural dominado por gramíneas y arbustos, y porque ofrecían un conjunto de bienes fundamentales a los viajeros.

Sin embargo, estas referencias no permiten asumir que la población de $S$. humboldtiana era continua y extensa; la mención puede indicar no tanto la abundancia como la escasez general de árboles y de esta especie en particular. 
El norte de la Patagonia representa el límite austral de la distribución natural de $S$. humboldtiana, cuyo clima árido, mediterráneo y continental restringiría su desempeño ecológico. A esta limitación, se le superpondrían los efectos negativos de la invasión biológica y la hibridación, el uso forestal no sustentable y la transformación riparia causada por la urbanización, la agricultura, la extracción de áridos y las represas hidroeléctricas. Estos factores habrían promovido una disminución poblacional de $S$. humboldtiana en los bordes de los cauces patagónicos hasta convertirla en una población relictual, cuya conservación está regionalmente amenazada.

Inequívocamente, $S$. humboldtiana está confinado a un hábitat muy restringido y fragmentado, cuyo tamaño probablemente es mucho más pequeño del que ocurrió en el pasado reciente. Esta situación justifica la inclusión de la especie al marco que brindan las leyes nacional y provincial de protección ambiental de bosques naturales. Sin embargo, el proceso de degradación actual además demanda programas de desarrollo y conservación en los cuales las dimensiones social y ecosistémica sean claves.

\section{RECONOCIMIENTOS}

Este proyecto fue financiado por la Universidad Nacional del Comahue y el Proyecto de Conservación para el Ordenamiento Territorial de los Bosques Nativos de Neuquén. Se agradece a los pobladores y el al Gobierno de Bajada del Agrio y Quili Malal, y a la Autoridad Interjurisdiccional de Cuencas por colaborar en este estudio. A. Martínez (Instituto Nacional de Tecnología Agropecuaria), F. Hidalgo (Departamento de Desarrollo Forestal de Neuquén), L. Dezzotti (Universidad Nacional de Córdoba) y G. Beltrami asistieron durante diferentes instancias del proyecto.

\section{REFERENCIAS}

AIC, 2020. Datos meteorológicos de la estación Bajada del Agrio durante 2000-2019. Autoridad Interjurisdiccional de Cuencas. Neuquén. 2 p.

Argus, G.W., 1997. Infrageneric classification of Salix (Salicaceae) in the New World. Systematic Botany Monographs 52: 1-121.

Barstow, M., 2018. Salix humboldtiana. The IUCN red list of threatened species. Disponible en: https://dx.doi.org/10.2305/IUCN.UK.2018-1.RLTS.T61960315A61960317.en. Acceso agosto 2020.

Batis, A.l.; Alcocer, M.I.; Gual, M.; Sánchez, C.; Vázquez-Yanes, C., 1999. Árboles y arbustos nativos potencialmente valiosos para la restauración ecológica y la reforestación. Instituto de Ecología, UNAM CONABIO. 15 p.

Belov, $\quad$ M., 2009.2 humboldtiana. Disponible http://www.chileflora.com/Florachilena/FloraSpanish/HighResPages/ SH0571.htm. Acceso agosto 2020.

Bendini, M. y Alemany, C., 2004. Crianceros y chacareros en la Patagonia. Cuaderno de GESA 5. Editorial La Colmena. $220 \mathrm{p}$.

Birken, A.S. y Cooper, D.J., 2006. Processes of Tamarix invasion and floodplain development along the lower Green River, Utah. Ecological Applications 16(3): 1103-1120.

Boelcke, O., 1986. Plantas vasculares de la Argentina. Nativas y Exóticas. Editorial Hemisferio Sur. 75 p. 
Borodowski, E.D. y Suárez, R.O., 2004. El cultivo de álamos y sauces: su historia en el delta del Paraná. SAGPyA Forestal 32: 5-13.

Bozzi, J.A.; Marchelli, P. y Gallo, L., 2014. Sauce criollo: una especie nativa amenazada en Patagonia. INTA Presencia 62: 29-33.

Brea, M.; Zucol, A. y Mazzanti, D., 2001. Determinación de combustibles vegetales en cueva El Abra, provincia de Buenos Aires. Actas XIV Congreso Nacional de Arqueología Argentina. p. 693-700.

Brea, M.; Mazzanti, D. y Martínez, G., 2014. Selección y uso de los recursos madereros en cazadoresrecolectores de la transición Pleistoceno-Holoceno y Holoceno medio, sierras de Tandilia oriental, Argentina. Revista Museo Argentino Ciencias Naturales 16(2): 129-141.

Budde, K.B.; Gallo, L.; Marchelli, P.; Mosner, E.; Liepelt, S.; Ziegenhagen, B y Leyer, I., 2011. Wide spread invasion without sexual reproduction? A case study on European Willows in Patagonia, Argentina. Biological Invasions 13: 45-54.

Cabrera, A.L., 1953. Esquema fitogeográfico de la República Argentina. Revista del Museo de La Plata (Sección Botánica) 8(33): 88-168.

Cailliez, F., 1980. Estimación del volumen forestal y predicción del rendimiento. Informe FAO 22/1. 92 p.

Casamiquela, R.M., 1985. Bosquejo de una etnología de la provincia de Río Negro. Edición Fundación Ameghino. 135 p.

Caso, S.L.; Neiff, J.J. y Neiff, A.P., 2010. Ecological responses of two pioneer species to a hydrological connectivity gradient in riparian forests of the lower Paraná river. Plant Ecology 209: 167-177.

Claraz, G., 2008. Viaje al río Chubut: aspectos naturalísticos y etnológicos (1865-1866). Ediciones Continente. $288 \mathrm{p}$.

Colobig, M., 2012. Estudios paleobotánicos del sitio 1, localidad arqueológica Lobería I, Buenos Aires, Argentina: aproximación al manejo de recursos vegetales. Relaciones de la Sociedad Argentina de Antropología 37(1): 137158.

Cremer, K.W., 2003. Introduced Willows can become invasive pests in Australia. Biodiversity 4: 17-24.

Darwin, C., 2009. Viaje de un naturalista alrededor del mundo. Miraguano Ediciones. 464 p.

Datri, L.A.; Maddio, R.; Faggi, A.M. y Gallo, L., 2013. Bosques ribereños y su relación con regímenes hidrológicos en el norte patagónico. Revista de la Asociación Argentina de Ecología de Paisajes 4(2): $245-259$.

DEEDI, 2020. Weeds of Australia. Department of Employment, Economic Development and Innovation. Disponible en: https://keyserver.lucidcentral.org/weeds/data/media/Html/salix_humboldtiana_pyramidalis.htm. Acceso agosto 2020 .

de la Cruz, L., 1806. Diario de viaje. Archivo General de la Nación, Sala IX, División Colonia, Legajo 39-5-5, Expediente $N^{\circ}$ 1, Archivo Nacional Histórico de Chile. Volumen 934.

de Martonne, E., 1926. Regions of interior-basin drainage. Geographical Review 17(3): 397-414.

Di Sapio, O. y Gattuso, M., 1994. Caracteres morfoanatómicos de diferentes cortezas de la medicina folklórica argentina. I. Salix humboldtiana Willd. (Salicaceae). Dominguezia 10(1): 18-26.

d'Orbigny, A., 1945. Viaje a la América meridional. Tomo II. Editorial Futuro. 569 p.

DPEC, 2010. Estadísticas de la provincia de Neuquén. Dirección Provincial de Estadística y Censos de Neuquén. Disponible en: https://www.estadisticaneuquen.gob.ar. Acceso agosto 2020. 
EMHIDRO, 2013. Apuntes sobre el Aprovechamiento Multipropósito Chihuido I. Emprendimientos Hidroeléctricos Sociedad del Estado Provincial del Neuquén. 31 p.

Fritts, H.C., 1982. An overview of dendroclimatic techniques, procedures and prospects, En: Hughes, M.K., Kelly, P.M., Pilcher, J.R., LaMarche, V.C., (eds.). Climate from tree rings. Cambridge University Press. pp. 191-197.

Hauenstein, E.; Peña-Cortés, F.; González, M. y Schlatter, R., 2005. Nuevos limites para la distribución de Salix humboldtiana Willd., Salicaceae, en Chile. Gayana Botánica 62(1): 44-46.

Hauman, L., 1913. Étude phytogéographique de la región du Rio Negro inférieur (Republique Argentine). Anales del Museo Nacional de Historia Natural 24: 289-444.

Hunziker, J.H., 1992. The origin of the hybrid triploid Willows cultivated in Argentina. Silvae Genetica 11: 151153.

ISO, 2012. ISO 3129: Wood sampling methods and general requirements for physical and mechanical testing of small clear wood specimens. International Organization for Standardization.

ISO, 2014a. ISO 13061-1: Physical and mechanical properties of wood - Test methods for small clear wood specimens - Part 1: Determination of moisture content for physical and mechanical tests. International Organization for Standardization.

ISO, 2014b. ISO 13061-1: Physical and mechanical properties of wood - Test methods for small clear wood specimens -Part 2: Determination of density for physical and mechanical tests. International Organization for Standardization.

Jost, L.; Chao, A. y Chazdon, R.L., 2010. Compositional similarity and $\beta$ (beta) diversity. En: Magurran, A.E., McGill, B.J., (eds.). Biological diversity: frontiers in measurement and asssessment. Oxford University Press. $p$. 66-84.

Kent, M., 2011. Vegetation description and data analysis: a practical approach. Wiley-Blackwell. 428 p.

Kuzovkina, Y.A.; Weih, M.; Romero, M.A.; Charles, J.; Hust, S.; Mclvor, I.; Karp, A.; Trybush, S.;Labrecque, M.; Teodorescu, T.I.; Singh, N.B.; Smart, L.B. y Volk, T.A., 2008. Salix: botany and global horticulture. Horticultural Reviews 34: 447-489.

Larroulet, A.; Résico, C.; Arbeletche, G.; Benmuyal, L. y Bejar, W., 2011. Usos no madereros de Salix humboldtiana. Actas III Congreso Internacional de Salicáceas en Argentina. 9 p.

Lewis, J.P. y Franceschi, E.A., 1979. Notas sobre la dinámica de la vegetación del valle del río Paraná. Ecosur 6(12): 145-163.

Liotta, J., 2001. Rasgos biológicos de Salix humboldtiana Willd. y régimen de pulsos de inundación. Interciencia 26(9): 397-403.

López, V.F., 1886. Acuerdos del extinguido Cabildo de Buenos Aires publicados bajo la dirección del Doctor Vicente F. López por encargo de la Municipalidad de la Capital. Imprenta Pablo E. Coni e Hijos. 490 p.

Lorentz, P. y Niederlein, G., 1883. Informe oficial de la comisión científica agregada al Estado Mayor General de la Expedición al Río Negro (Patagonia). II Botánica. Enumeración sistemática de las plantas colectadas durante la expedición. Academia Nacional de Ciencias de Córdoba. 178 p.

LPN, 2020. Ley 2780. Legislatura de la Provincia de Neuquén. Disponible en: http://digesto.smandes.gov.ar. Acceso agosto 2020.

MJDH, 2020. Ley 26331. Ministerio de Justicia y Derechos Humanos de la Nación. Disponible en: http://servicios.infoleg.gob.ar/infoleglnternet/anexos/135000139999/136125/norma.htm\#: :text=ARTICULO\%201\%C2\%BA\%20\%

E2\%80\%94\%20La\%20presente\%20ley,\%C3\%A9stos\%20brindan\%20a\%20la\%20sociedad. Acceso agosto 2020. 
Moggridge, H.L. y Gurnell, A.M., 2009. Controls on the sexual and asexual regeneration of Salicaceae along a highly dynamic, braided river system. Aquatic Science 71: 305-317.

Molina, J., 1805. Diario que debo formar desde la Ciudad de Sn. Bartolomé de Chillan, atravesando por las Cordilleras de Alico, hasta llegar a la Ciudad de Buenos Ayres, y desde esta de mi regreso hasta llegar a la Ciudad de la Concepción, en la que debo dar cuenta de la Comisión que el Sr. Governador Yntendente se digno darme para la exploración del Camino a Buenos Ayres. Sala IX, 39-5-5, Legajo 1. Archivo General de la Nación.

Mollo, N. y Vignolo, E., 2010. Contribuciones del diario de viaje de Justo Molina a la toponimia aborigen del norte neuquino. Sociedades de Paisajes Áridos y Semi-Áridos 2(3): 117-131.

Moreno, F.P., 1969. Viaje a la Patagonia Austral 1876-1877. Ediciones Solar Hachette. 407 p.

Natale, E.; Gaskin, J.; Zalba, S.M.;Ceballos, M. y Reinoso, H.E., 2008 Especies del género Tamarix (Tamaricaceae) invadiendo ambientes naturales y seminaturales en Argentina. Boletín Sociedad Argentina de Botánica 43(1-2): 137-145.

Natale, E.; Zalba, M.S.,;Reinoso, H. y Damilano, G., 2012. Assessing invasion process through pathway and vector analysis: case of Saltcedar (Tamarix spp.). Management of Biological Invasions 3: 37-44.

Natale, E.; Reinoso, H.E.; Andreo, V. y Zalba, S.M., 2018. Mapeo del riesgo: prioridades para prevenir el establecimiento de tamariscos invasores. Ecología Austral 28: 81-92.

Oyarzábal, M.; Clavijo, J.; Oakley, L.; Biganzoli, F.: Tognetti, P.; Barberis, I.; Maturo, H.; Aragón, R.; Campanello, P.; Prado, D.; Oesterheld, M. y León, R., 2018. Unidades de vegetación de la Argentina. Ecología Austral 28: 40-63.

Parolin, P.; Oliveira, A.C.; Piedade, M.T.F.; Wittmann, F. y Junk, W.J., 2002. Pioneer trees in Amazonian floodplains: three key species form monospecific stands in different habitats. Folia Geobotanica 37: 225-238.

Peel, M.C.; Finlayson, B.L. y McMahon, T.A., 2007. Updated world map of the Köppen-Geiger climate classification. Hydrological Earth System Science 11: 1633-1644.

Pereyra, F. y Bouza, P., 2019. Soils from the Patagonian region. En: Rubio, G., Lavado, R., Pereyra, F. (eds.). The soils of Argentina. World Soils Book Series. Springer. p. 101-121.

Prates, L., 2009. El uso de recursos por los cazadores-recolectores posthispánicos de Patagonia Continental y su importancia Arqueológica. Relaciones de la Sociedad Argentina de Antropología 34: 201-229.

Rodríguez, M., 2000. Woody plant species used during the Archaic period in the Southern Argentine Puna. Archaeobotany of Quebrada Seca 3. Journal of Arcaheological Science 27: 341-361.

Rodríguez, R.; Matthei, O. y Quezada, M., 1983. Flora arbórea de Chile. Editorial de la Universidad de Concepción. 408 p.

Roig, F.A., 1955. El sauce criollo (Salix humboldtiana Willd.) en los ríos San Juan y Desagüadero. Revista de la Facultad de Ciencias Agrarias 5(1): 51-56.

Scaramuzzino, R.; Bardi, J.; D’Alfonso, C. y Villamil, C., 2017. Dificultades en la reconstrucción de la distribución de Salix humboldtiana Willd. basado en fuentes documentales en el centro-sur de la provincia de Buenos Aires. Revista de la Asociación Argentina de Ecología de Paisajes 7(1): 1-15.

SEN, 2020. Secretaría de Energía de la Nación. Disponible en: http://www.energia.gob.ar/contenidos/ verpagina.php?idpagina=3622. Acceso agosto 2020 .

Shafroth, P.B.; Scott, M.L. y Friedman, J.M., 1994. Establishment, sex structure and breeding system of an exotic riparian Willow, Salix x rubens. American Midland Naturalist 132: 159-172.

Thomas, L.K.; Tölle, L.; Ziegenhagen, B. y Leyer, I., 2012. Are vegetative reproduction capacities the cause of 
widespread invasion of Eurasian Salicaceae in Patagonian river landscapes? PLoS One 7(12): 652.

Thomas, L.K. y Leyer, I., 2014. Age structure, growth performance and composition of native and invasive Salicaceae in Patagonia. Plant Ecology 215(9): 1047-1056.

Thornthwaite, C.W. y Matter, J.R., 1957. Instructions and tables for computing potential evapotranspiration and the water balance. Climatology 10(3): 185-311.

Triana, M.A., 2001. Factor de conversión de unidades "estéreo" a $\mathrm{m}^{3}$ para cuantificar volúmenes de leña: un estudio de caso en el sur de Chile. Colombia Forestal 7(14): 25-32.

Villarino, B., 1837. Diario del piloto de la Real Armada, don Basilio Villarino, del reconocimiento que hizo del río Negro, en la costa oriental de Patagonia, el año de 1782. Editorial Impresiones del Estado. 131 p. 